\title{
Boundedness of Calderón-Zygmund Operator and Their Commutator on Herz Spaces with Variable Exponent
}

\author{
Omer Abdalrhman ${ }^{1,2^{*}}$, Afif Abdalmonem ${ }^{1,3}$, Shuangping Tao ${ }^{1}$ \\ ${ }^{1}$ College of Mathematics and Statistics, Northwest Normal University, Lanzhou, China \\ ${ }^{2}$ College of Education, Shendi University, Shendi, River Nile State, Sudan \\ ${ }^{3}$ Faculty of Science, University of Dalanj, Dalanj, South kordofan, Sudan \\ Email: ^humoora@gmail.com, taosp@nwnu.edu.cn, afeefy86@gmail.com
}

How to cite this paper: Abdalrhman, O., Abdalmonem, A. and Tao, S.P. (2017) Boundedness of Calderón-Zygmund Operator and Their Commutator on Herz Spaces with Variable Exponent. Applied Mathematics, 8, 428-443.

https://doi.org/10.4236/am.2017.84035

Received: March 13, 2017

Accepted: April 17, 2017

Published: April 20, 2017

Copyright $\odot 2017$ by authors and Scientific Research Publishing Inc. This work is licensed under the Creative Commons Attribution International License (CC BY 4.0).

http://creativecommons.org/licenses/by/4.0/

\begin{abstract}
The aim of this paper is to study the boundedness of Calderón-Zygmund operator and their commutator on Herz Spaces with two variable exponents $p(),. q($.$) . By applying the properties of the Lebesgue spaces with variable$ exponent, the boundedness of the Calderón-Zygmund operator and the commutator generated by BMO function and Calderón-Zygmund operator is obtained on Herz space.
\end{abstract}

\section{Keywords}

Calderón-Zygmund Operator, Commutator, Herz Spaces with Variable Exponent, BMO Spaces

\section{Introduction}

Definition 1.1. Let $T$ be a bounded linear operator from $S\left(\mathbb{R}^{n}\right)$ to $S^{\prime}\left(\mathbb{R}^{n}\right)$ (see [1], [2]). $T$ is called a standard operator if $T$ satisfies the following conditions:

1) $T$ extends to a bounded linear operator on $L^{2}\left(\mathbb{R}^{n}\right)$.

2) There exists a function $K(x, y)$ defined by $\left\{(x, y) \in\left(\mathbb{R}^{n}\right) \times\left(\mathbb{R}^{n}\right) ; x \neq y\right\}$ satisfies

$$
|K(x, y)| \leq C /|x-y|^{n},
$$

where $C>0$.

3) $\langle T f, g\rangle=\int_{\left(\mathbb{R}^{n}\right)} \int_{\left(\mathbb{R}^{n}\right)} K(x, y) f(y) g(x) \mathrm{d} x \mathrm{~d} y$, for $f, g \in S\left(\mathbb{R}^{n}\right)$ with $\operatorname{supp}(f) \cap \operatorname{supp}(g)=\varnothing$ 
A standard operator $T$ is called a $\gamma$-Calderón-Zygmund operator if $K$ is a standard kernel satisfies:

$$
\begin{aligned}
& \qquad|K(x, y)-K(z, y)| \leq C|x-z|^{\gamma} /|x-y|^{n+\gamma} ; \\
& |K(y, x)-K(y, z)| \leq C|x-z|^{\gamma} /|x-y|^{n+\gamma}, \\
& \text { if }|x-z|<\frac{1}{2}|x-y| \text { for some } 0<\gamma \leq 1 .
\end{aligned}
$$

The bounded mean oscillation BMO space and BMO norm are defined, respectively, by

$$
\begin{gathered}
B M O\left(\mathbb{R}^{n}\right)=\left\{b \in L_{\text {loc }}^{1}\left(\mathbb{R}^{n}\right):\|b\|_{B M O\left(\mathbb{R}^{n}\right)}<\infty\right\}, \\
\|b\|_{B M O\left(\mathbb{R}^{n}\right)}=\sup _{B: \text { ball }} 1 /|B| \int_{B}\left|b(x)-b_{B}\right| \mathrm{d} x .
\end{gathered}
$$

The commutator of the Calderón-Zygmund operator is defined by

$$
[b, T] f(x)=b(x) T f(x)-T(b f)(x) .
$$

In 1983, J.-L. Jouné proved $\gamma$-Calderón-Zygmund operator is bounded on $L^{p}\left(\mathbb{R}^{n}\right)$ in [3]. Coifman, Rochberg and Weiss proved that commutator [b,T] is bounded on $L^{p}\left(\mathbb{R}^{n}\right)(1<p<1)$ (see [4]).

Kovácik and Rákosník introduced Lebesgue spaces and Sobolev spaces with variable exponents (see [5]). The function spaces with variable exponent has been recently obtained an increasing interest by a number of authors since many applications are found in many different fields, for example, in fluid dynamics (see [6]), image restoration (see [7] [8] [9]) and differential equations.

Herz spaces play an important role in harmonic analysis. After they were introduced in [10], the boundedness of some operators and some characterizations of Herz spaces with variable exponents were studied extensively (see [11]-[16]). In 2015, Wang and Tao introduced the Herz spaces with two variable exponents $p(),. q($.$) , and studied the parameterized Littlewood-Paley operators$ and their commutators on Herz spaces with variable exponents in [17].

In this paper, we will discuss the boundedness of the Calderón-Zygmund operator $T$ and their commutator $[b, T]$ are bounded on Herz spaces with two variable exponents $p(),. q($.$) .$

\section{Definitions of Function Spaces with Variable Exponent}

In this section we recall some definitions. Let $\Omega$ be a measurable set in $\mathbb{R}^{n}$ with $|\Omega|>0$. We firstly recall the definition of the Lebesgue spaces with variable exponent.

Definition 2.1. [5] Let $p(\cdot): \Omega \rightarrow[1, \infty)$ be a measurable function. The Lebesgue space with variable exponent $L^{p(\cdot)}(\Omega)$ is defined by

$L^{p(\cdot)}(\Omega)=\left\{f\right.$ is measurable $: \int_{\Omega}\left(\frac{|f(x)|}{\eta}\right)^{p(x)} \mathrm{d} x<\infty$ for some constant $\left.\eta>0\right\}$.

For all compact $K \subset \Omega$, the space $L_{\text {loc }}^{p(\cdot)}(\Omega)$ is defined by 


$$
L_{\text {loc }}^{p(\cdot)}(\Omega)=\left\{f \text { is measurable }: f \in L^{p(\cdot)}(K)\right\} .
$$

The Lebesgue spaces $L^{p(\cdot)}(\Omega)$ is a Banach spaces with the norm defined by

$$
\|f\|_{L^{p(\cdot)}(\Omega)}=\inf \left\{\eta>0: \int_{\Omega}\left(\frac{|f(x)|}{\eta}\right)^{p(x)} \mathrm{d} x \leq 1\right\} .
$$

We denote $p_{-}=e s s i n f\{p(x): x \in \Omega\}, p_{+}=e s s \sup \{p(x): x \in \Omega\}$. Then $\mathcal{P}(\Omega)$ consists of all $p(\cdot)$ satisfying $p_{-}>1$ and $p_{+}<\infty$. Let $M$ be the Hardy-Littlewood maximal operator. We denote $\mathcal{B}(\Omega)$ to be the set of all function $p(\cdot) \in \mathcal{P}(\Omega)$ satisfying the $M$ is bounded on $L^{p(\cdot)}(\Omega)$.

Definition 2.2. [18] Let $p(\cdot), q(\cdot) \in \mathcal{P}(\Omega)$. The mixed Lebesgue sequence space with variable exponent $\ell^{q(\cdot)}\left(L^{p(\cdot)}\right)$ is the collection of all sequences $\left\{f_{j}\right\}_{j=0}^{\infty}$ of the measurable functions on $\mathbb{R}^{n}$ such that

$$
\begin{gathered}
\left\|\left\{f_{j}\right\}_{j=0}^{\infty}\right\|_{\ell^{q(\cdot)}\left(L^{p(\cdot)}\right)}=\inf \left\{\eta>0: Q_{\ell^{q(\cdot)}\left(L^{p(\cdot)}\right)}\left(\left\{\frac{f_{j}}{\zeta}\right\}_{j=0}^{\infty}\right) \leq 1\right\}<\infty, \\
\left.Q_{\ell^{q(\cdot)}\left(L^{p(\cdot)}\right)}\left(\left\{f_{j}\right\}_{j=0}^{\infty}\right)=\sum_{j=0}^{\infty} \inf \left\{\zeta_{j}>0 ; \int_{R^{n}} \frac{\left|f_{j}(x)\right|}{\frac{1}{\zeta_{j}^{q(x)}}}\right)^{p(x)} \mathrm{d} x \leq 1\right\} .
\end{gathered}
$$

Let $B_{k}=\left\{x \in \mathbb{R}^{n}:|x| \leq 2^{k}\right\}, C_{k}=B_{k} \backslash B_{k-1}, \chi_{k}=\chi_{C_{k}}, k \in \mathbb{Z}$, for $q_{+}<\infty$, we have that

$$
Q_{\ell^{q(\cdot)}\left(L^{p(\cdot)}\right)}\left(\left\{f_{j}\right\}_{j=0}^{\infty}\right)=\sum_{j=0}^{\infty}\left\|\left|f_{j}\right|^{q(\cdot)}\right\|_{L^{q(\cdot)}} \cdot
$$

Let $B_{k}=\left\{x \in \mathbb{R}^{n}:|x| \leq 2^{k}\right\}, C_{k}=B_{k} \backslash B_{k-1}, \chi_{k}=\chi_{C_{k}}, k \in \mathbb{Z}$.

Definition 2.3. [17] Let $\alpha \in \mathbb{R}^{n}, q(\cdot), p(\cdot) \in \mathcal{P}\left(\mathbb{R}^{n}\right)$. The homogeneous Herz space with variable exponent $\dot{K}_{p(\cdot)}^{\alpha, q(\cdot)}\left(\mathbb{R}^{n}\right)$ is defined by

$$
\dot{K}_{p(\cdot)}^{\alpha, q(\cdot)}\left(\mathbb{R}^{n}\right)=\left\{f \in L_{\text {loc }}^{p(\cdot)}\left(\mathbb{R}^{n} \backslash\{0\}\right):\|f\|_{\dot{K}_{p(\cdot)}^{\alpha, q(\cdot)}\left(\mathbb{R}^{n}\right)}<\infty\right\} .
$$

Equipped the norm

$$
\begin{aligned}
& \|f\|_{\dot{K}_{P(\cdot)}^{\alpha, q(\cdot)}\left(\mathbb{R}^{n}\right)}=\left\|\left\{2^{k \alpha}\left|f \chi_{k}\right|\right\}_{k=0}^{\infty}\right\|_{l^{q(\cdot)}\left(L^{p(\cdot)}\right)} \\
& =\inf \left\{\eta>0: \sum_{k=-\infty}^{\infty}\left\|\left(\frac{2^{k \alpha}\left|f \chi_{k}\right|}{\eta}\right)^{q(\cdot)}\right\|_{\frac{p(\cdot)}{q^{(\cdot)}}} \leq 1\right\} .
\end{aligned}
$$

Remark 2.1. [17] Let $q_{1}(\cdot), q_{2}(\cdot) \in \mathcal{P}\left(\mathbb{R}^{n}\right)$ satisfying $\left(q_{1}\right)_{+} \leq\left(q_{2}\right)_{+}$and satisfy the following results:

1) $\dot{K}_{p(\cdot)}^{\alpha, q_{1}(\cdot)}\left(\mathbb{R}^{n}\right) \subset \dot{K}_{p(\cdot)}^{\alpha, q_{2}(\cdot)}\left(\mathbb{R}^{n}\right)$.

2) If $\frac{q_{2}(\cdot)}{q_{1}(\cdot)} \in \mathcal{P}\left(\mathbb{R}^{n}\right)$ and $\frac{q_{2}(\cdot)}{q_{1}(\cdot)} \geq 1$. For any $f \in \dot{K}_{p(\cdot)}^{\alpha, q(\cdot)}\left(\mathbb{R}^{n}\right)$, by using 
Lemma 3.7 and Remark 2.2, we have

$$
\begin{aligned}
\sum_{k=-\infty}^{\infty}\left\|\left(\frac{2^{k \alpha}\left|f \chi_{k}\right|}{\eta}\right)^{q_{2}(\cdot)}\right\|_{\frac{p(\cdot)}{q_{2}(\cdot)}} & \leq \sum_{k=-\infty}^{\infty}\left\|\left(\frac{2^{k \alpha}\left|f \chi_{k}\right|}{\eta}\right)^{q_{1}(\cdot)}\right\|_{\frac{p(\cdot)}{q_{1}(\cdot)}}^{p_{v}} \\
& \leq\left\{\sum_{k=-\infty}^{\infty}\left\|\left(\frac{2^{k \alpha}\left|f \chi_{k}\right|}{\eta}\right)^{q_{1}(\cdot)}\right\|_{\frac{p(\cdot)}{p_{h}}}^{p_{q_{1}(\cdot)}}\right\}^{p_{*}} \leq 1 .
\end{aligned}
$$

where

$$
\begin{gathered}
p_{v}=\left\{\begin{array}{l}
\left(\frac{q_{2}(\cdot)}{q_{1}(\cdot)}\right)_{-}, \frac{2^{k \alpha}\left|f \chi_{k}\right|}{\eta} \leq 1, \\
\left(\frac{q_{2}(\cdot)}{q_{1}(\cdot)}\right)_{+}, \frac{2^{k \alpha}\left|f \chi_{k}\right|}{\eta}>1 .
\end{array}\right. \\
p_{*}=\left\{\begin{array}{l}
\min _{v \in \mathbb{N}} p_{v}, \sum_{v=0}^{\infty} a_{v} \leq 1, \\
\max _{v \in \mathbb{N}} p_{v}, \sum_{v=0}^{\infty} a_{v}>1 .
\end{array}\right.
\end{gathered}
$$

This implies that $\dot{K}_{p(\cdot)}^{\alpha, q_{1}(\cdot)}\left(\mathbb{R}^{n}\right) \subset \dot{K}_{p(\cdot)}^{\alpha, q_{2}(\cdot)}\left(\mathbb{R}^{n}\right)$.

Remark 2.2. Let $v \in \mathbb{N}, a_{v} \geq 0,1 \leq p_{v}<\infty$. Then we have

$$
\sum_{v=0}^{\infty} a_{v} \leq\left(\sum_{v=0}^{\infty} a_{h}\right)^{p_{*}},
$$

where

$$
p_{*}=\left\{\begin{array}{l}
\min _{v \in \mathbb{N}} p_{v}, \sum_{v=0}^{\infty} a_{v} \leq 1, \\
\max _{v \in \mathbb{N}} p_{v}, \sum_{v=0}^{\infty} a_{v}>1 .
\end{array}\right.
$$

\section{Properties and Lemmas of Variable Exponent}

In this section, we recall some properties and some lemmas of variable exponent belonging to the class $\mathcal{B}\left(\mathbb{R}^{n}\right)$.

Proposition 3.1. [19] If $p(\cdot) \in \mathcal{P}\left(\mathbb{R}^{n}\right)$ satisfies

$$
\begin{gathered}
|p(x)-p(y)| \leq \frac{-C}{\log (|x-y|)},|x-y| \leq 1 / 2 ; \\
|p(x)-p(y)| \leq \frac{C}{\log (e+|x|)},|y| \geq|x| .
\end{gathered}
$$

Hence we have $p(\cdot) \in \mathcal{B}\left(\mathbb{R}^{n}\right)$.

Lemma 3.1. [5] Given $p(\cdot): \mathbb{R}^{n} \rightarrow[1, \infty)$ have that for all functions $f$ and $g$

$$
\int_{\mathbb{R}^{n}}|f(x) g(x)| \mathrm{d} x \leq C\|f\|_{L^{p(\cdot)}\left(\mathbb{R}^{n}\right)}\|g\|_{L^{p^{\prime} \cdot(\cdot)}\left(\mathbb{R}^{n}\right)} \cdot
$$

where $C_{p}=1+\frac{1}{p_{-}}-\frac{1}{p_{+}}$. 
Lemma 3.2. [5] Suppose that $p(\cdot), p_{1}(\cdot), p_{2}(\cdot) \in \mathcal{P}\left(\mathbb{R}^{n}\right)$, for any $f \in L^{p_{1}(\cdot)}\left(\mathbb{R}^{n}\right), g \in L^{p_{2}(\cdot)}\left(\mathbb{R}^{n}\right)$, when $\frac{1}{p(\cdot)}=\frac{1}{p_{2}(\cdot)}+\frac{1}{p_{1}(\cdot)}$, we get

$$
\|f(x) g(x)\|_{L^{p(\cdot)}\left(\mathbb{R}^{n}\right)} \leq C\|g(x)\|_{L^{p_{2}}\left(\mathbb{R}^{n}\right)}\|f(x)\|_{L^{p_{1} \cdot(\cdot)}\left(\mathbb{R}^{n}\right)},
$$

where $C_{p_{1}, p_{2}}=\left[1+\frac{1}{p_{1-}}-\frac{1}{p_{1+}}\right]^{\frac{1}{p_{-}}}$.

Proposition 3.2. [20] Let $p(\cdot) \in \mathcal{B}\left(\mathbb{R}^{n}\right)$ and $T$ be a Calderón-Zygmund operator. Then we have

$$
\|T f\|_{L^{(\cdot)}\left(\mathbb{R}^{n}\right)} \leq C\|f\|_{L^{p(\cdot)}\left(\mathbb{R}^{n}\right)} \cdot
$$

Lemma 3.3. [20] Let $p(\cdot) \in \mathcal{B}\left(\mathbb{R}^{n}\right), b \in \mathrm{BMO}$ function and $T$ be a Calderón - Zygmund operator.Then

$$
\|[b, T] f\|_{L^{p \cdot(\cdot)}\left(\mathbb{R}^{n}\right)} \leq C\|b\|_{\mathrm{BMo}\left(\mathbb{R}^{n}\right)}\|f\|_{L^{p(\cdot)}\left(\mathbb{R}^{n}\right)}
$$

Lemma 3.4. [11] Let $b \in \operatorname{BMO}\left(\mathbb{R}^{n}\right)$. If $i, j \in \mathbb{Z}$ with $i<j$, then we have

1. $C^{-1}\|b\|_{\mathrm{BMO}\left(\mathbb{R}^{n}\right)} \leq \sup _{B} \frac{1}{\left\|\chi_{B}\right\|_{L^{(\cdot)}\left(\mathbb{R}^{n}\right)}}\left\|\left(b-b_{B}\right) \chi_{B}\right\|_{L^{p(\cdot)}\left(\mathbb{R}^{n}\right)} \leq C\|b\|_{\mathrm{BMO}\left(\mathbb{R}^{n}\right)}$.

2. $\left\|\left(b-b_{B_{i}}\right) \chi_{B_{j}}\right\|_{L^{q(\cdot)}\left(\mathbb{R}^{n}\right)} \leq C(j-i)\|b\|_{B_{M O}\left(\mathbb{R}^{n}\right)}\left\|\chi_{B_{j}}\right\|_{L^{q(\cdot)}\left(\mathbb{R}^{n}\right)}$.

Lemma 3.5. [21] Let $p_{u}(\cdot) \in \mathcal{B}\left(\mathbb{R}^{n}\right)(u=1,2)$, then there exist constants $0<l_{u 1}, l_{u 2}<1$, and $C>0$ such that for all balls $B \subset \mathbb{R}^{n}$ and all measurable subset $R \subset B$,

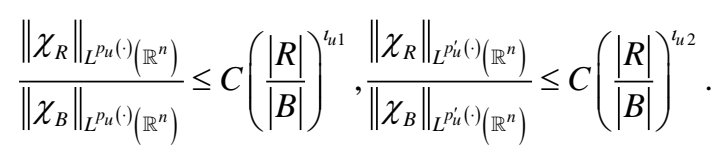

Lemma 3.6. [11] If $p(\cdot) \in \mathcal{B}\left(\mathbb{R}^{n}\right)$, there exist a constant $C>0$ such that for any balls $B$ in $\mathbb{R}^{n}$, we have

$$
\frac{1}{|B|}\left\|\chi_{B}\right\|_{L^{(\cdot)}\left(\mathbb{R}^{n}\right)}\left\|\chi_{B}\right\|_{L^{p^{(\cdot)}\left(\mathbb{R}^{n}\right)}} \leq C .
$$

Lemma 3.7. [17] Suppose that $p(\cdot), q(\cdot) \in \mathcal{P}\left(\mathcal{B}^{n}\right)$. If $f \in L^{p(\cdot) q(\cdot)}$, then

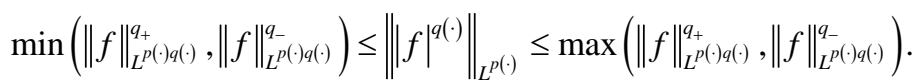

\section{The Main Theorems and Their Proofs}

Theorem 4.1. Suppose that $p_{1}(\cdot) \in \mathcal{B}\left(\mathbb{R}^{n}\right), q_{1}(\cdot), q_{2}(\cdot) \in \mathcal{P}\left(\mathbb{R}^{n}\right)$ with $\left(q_{2}\right)_{-} \geq\left(q_{1}\right)_{+}$. If $-n l_{12}<\alpha<n l_{11}$ with $l_{11}, l_{12}$ as defined in Lemma 3.5 , then the operator $T$ is bounded from $\dot{K}_{p_{1}(\cdot)}^{\alpha, q_{2}(\cdot)}\left(\mathbb{R}^{n}\right)$ to $\dot{K}_{p_{1}(\cdot)}^{\alpha, q_{1}(\cdot)}\left(\mathbb{R}^{n}\right)$.

Proof Let $h(x) \in \dot{K}_{p_{1}(\cdot)}^{\alpha, q_{1} \cdot(\cdot)}\left(\mathbb{R}^{n}\right)$. We write

$$
h(x)=\sum_{j=-\infty}^{\infty} h(x) \chi_{j}=\sum_{j=-\infty}^{\infty} h_{j}(x) .
$$


By Definition 2.3, we have

$$
\|T(h)\|_{\dot{K}_{p_{1}(\cdot)}^{\alpha, q_{2}(\cdot)\left(\mathbb{R}^{n}\right)}}=\inf \left\{\eta>0: \sum_{k=-\infty}^{\infty}\left\|\left(\frac{2^{k \alpha}\left|T(h) \chi_{k}\right|}{\eta}\right)^{q_{2}(\cdot)}\right\|_{\frac{p_{L^{2}(\cdot)}}{q_{2(\cdot)}}} \leq 1\right\} .
$$

Since

$$
\begin{aligned}
& \left\|\left(\frac{2^{k \alpha}\left|T(h) \chi_{k}\right|}{\eta}\right)^{q_{2}(\cdot)}\right\|_{L_{L^{\frac{p}{2}(\cdot)}}^{q_{2(\cdot)}}} \leq\left\|\left(\frac{2^{k \alpha}\left|\sum_{j=-\infty}^{\infty} T\left(h_{j}\right) \chi_{k}\right|}{\sum_{i=1}^{3} \eta_{1 i}}\right)^{q_{2}(\cdot)}\right\|_{\frac{p_{1}(\cdot)}{q_{2}(\cdot)}} \\
& \leq\left\|\left(\frac{2^{k \alpha}\left|\sum_{j=-\infty}^{k-2} T\left(h_{j}\right) \chi_{k}\right|}{\eta_{11}}\right)^{q_{2}(\cdot)}\right\|_{\frac{p_{1}(\cdot)}{L_{2(\cdot)}}} \\
& +\left\|\left(\frac{2^{k \alpha}\left|\sum_{j=k-2}^{k+2} T\left(h_{j}\right) \chi_{k}\right|}{\eta_{12}}\right)^{q_{2}(\cdot)}\right\|_{\frac{p_{1}(\cdot)}{q_{2(\cdot)}}} \\
& +\left\|\left(\frac{2^{k \alpha}\left|\sum_{j=k+2}^{\infty} T\left(h_{j}\right) \chi_{k}\right|}{\eta_{13}}\right)^{q_{2}(\cdot)}\right\|_{\frac{p_{1}(\cdot)}{L_{2}(\cdot)}},
\end{aligned}
$$

where

$$
\begin{aligned}
& \eta_{11}=\left\|\left\{2^{k \alpha}\left|\sum_{j=-\infty}^{k-2} T\left(h_{j}\right) \chi_{k}\right|\right\}_{k=-\infty}^{\infty}\right\|_{\ell^{q_{2}(\cdot)}\left(L^{p_{1}(\cdot)}\right)}, \\
& \eta_{12}=\left\|\left\{2^{k \alpha}\left|\sum_{j=k-2}^{k+2} T\left(h_{j}\right) \chi_{k}\right|\right\}_{k=-\infty}^{\infty}\right\| \|_{\ell^{q_{2}(\cdot)}\left(L^{p_{1}(\cdot)}\right)}, \\
& \eta_{13}=\left\|\left\{2^{k \alpha}\left|\sum_{j=k+2}^{\infty} T\left(h_{j}\right) \chi_{k}\right|\right\}_{k=-\infty}^{\infty}\right\| \|_{\ell^{q_{2}(\cdot)}\left(L^{p_{1}(\cdot)}\right)},
\end{aligned}
$$

and

$$
\eta=\sum_{i=1}^{3} \eta_{1 i}
$$

Thus,

$$
\sum_{k=-\infty}^{\infty}\left\|\left(\frac{2^{k \alpha}\left|T(h) \chi_{k}\right|}{\eta}\right)^{q_{2}(\cdot)}\right\|_{\frac{p_{1}(\cdot)}{q_{2(\cdot)}}} \leq C .
$$

We easily see that

$$
\|T(h)\|_{\dot{K}_{p_{1}(\cdot)}^{\alpha, q_{2}(\cdot)}\left(\mathbb{R}^{n}\right)} \leq C \eta=C \sum_{i=1}^{3} \eta_{1 i} .
$$

This implies that we only need to prove $\eta_{11}, \eta_{12}, \eta_{13} \leq C\|h\|_{\dot{K}_{p_{1}(\cdot)}^{\alpha, q_{1}(\cdot)}\left(\mathbb{R}^{n}\right)}$. Denote $\eta_{10}=\|h\|_{\dot{K}_{p_{1}(\cdot)}^{\alpha, q_{1} \cdot(\cdot)}\left(\mathbb{R}^{n}\right)} \cdot$ 
First, we consider $\eta_{12}$. By virtue of Lemma 3.7, we get

$$
\begin{aligned}
& \sum_{k=-\infty}^{\infty}\left\|\left(\frac{2^{k \alpha}\left|\sum_{j=k-2}^{k+2} T\left(h_{j}\right) \chi_{k}\right|}{\eta_{10}}\right)^{q_{2}(\cdot)}\right\|_{\frac{p_{1}(\cdot)}{q_{2}(\cdot)}} \\
& \leq \sum_{k=-\infty}^{\infty}\left\|\frac{2^{k \alpha} \mid \sum_{j=k-2}^{k+2} T\left(h_{j}\right) \chi_{k}}{\eta_{10}}\right\|_{L^{p_{1} \cdot(\cdot)}}^{\left(q_{2}^{1}\right)_{k}} \\
& \leq \sum_{k=-\infty}^{\infty}\left(\left\|\frac{2^{k \alpha} \mid \sum_{j=k-2}^{k+2} T\left(h_{j}\right) \chi_{k} \|}{\eta_{10}}\right\|_{L^{p_{1}(\cdot)}}\right)^{\left(q_{2}^{1}\right)_{k}},
\end{aligned}
$$

where,

$$
\left(q_{2}^{1}\right)_{k}=\left\{\begin{array}{l}
\left(q_{2}\right)_{-},\left\|\left(\frac{2^{k \alpha}\left|\sum_{j=k-2}^{k+2} T\left(h_{j}\right) \chi_{k}\right|}{\eta_{10}}\right)^{q_{2}(\cdot)}\right\|_{\frac{p_{1}(\cdot)}{q_{2}(\cdot)}} \leq 1, \\
\left(q_{2}\right)_{+},\left\|\frac{\left.2^{k \alpha}\left|\sum_{j=k-2}^{k+2} T\left(h_{j}\right) \chi_{k}\right|\right)^{q_{2}(\cdot)}}{\eta_{10}}\right\|_{\frac{p_{1}(\cdot)}{q_{2}(\cdot)}}>1 .
\end{array}\right.
$$

In the above, we use the Proposition 3.2 and Remark 2.2. Since

$$
\begin{aligned}
& h(x) \in \dot{K}_{p_{1}(\cdot)}^{\alpha, q_{1}(\cdot)}\left(\mathbb{R}^{n}\right) \text {, we have }\left\|\frac{2^{k \alpha}\left|h \chi_{k}\right|}{\eta_{10}}\right\|_{L^{p_{1}(\cdot)}} \leq 1 \text { and } \\
& \sum_{k=-\infty}^{\infty}\left\|\left(\frac{2^{k \alpha}\left|h \chi_{k}\right|}{\eta_{10}}\right)^{q_{1}(\cdot)}\right\|_{\frac{p_{1}(\cdot)}{L_{1}(\cdot)}} \leq 1 \text {, we get } \\
& \sum_{k=-\infty}^{\infty}\left\|\left(\frac{2^{k \alpha}\left|\sum_{j=k-2}^{k+2} T\left(h_{j}\right) \chi_{k}\right|}{\eta_{10}}\right)^{q_{2}(\cdot)}\right\|_{\frac{p_{1}(\cdot)}{q_{2}(\cdot)}} \\
& \leq C \sum_{k=-\infty}^{\infty}\left(\sum_{j=k-2}^{k+2}\left\|\frac{2^{k \alpha}\left|h_{j}\right|}{\eta_{10}}\right\|_{L^{p_{1}(\cdot)}}\right)^{\left(q_{2}^{1}\right)_{k}} \\
& \leq C \sum_{k=-\infty}^{\infty}\left\|\frac{2^{k \alpha} \mid h \chi_{k}}{\eta_{10}}\right\|_{L^{p_{1}(\cdot)}}^{\left(q_{2}^{1}\right)_{k}} \\
& \leq C \sum_{k=-\infty}^{\infty} \|\left(\frac{2^{k \alpha}\left|h \chi_{k}\right|}{\eta_{10}}\right)^{q_{1}(\cdot)} \mid \begin{array}{l}
\left(\frac{\left.q_{2}^{1}\right)_{k}}{\left(q_{1}\right)_{+}}\right. \\
\frac{p_{1} \cdot(\cdot)}{L^{1(\cdot)}}
\end{array} \\
& \leq C\left\{\sum_{k=-\infty}^{\infty}\left\|\left(\frac{2^{k \alpha}\left|h \chi_{k}\right|}{\eta_{10}}\right)^{q_{1}(\cdot)}\right\|_{\frac{p_{(-)}(\cdot)}{q_{q_{1}} \cdot()}}\right\}^{q_{*}} \\
& \leq C \text {. }
\end{aligned}
$$


Here $\left(p_{1}\right)_{+} \leq\left(p_{2}\right)_{-} \leq\left(q_{2}^{1}\right)_{k}$ and $q_{*}=\min _{k \in N} \frac{\left(q_{2}^{1}\right)_{k}}{\left(q_{1}\right)_{+}}$. That is

$$
\eta_{12} \leq C \eta_{10} \leq C\|h\|_{\dot{K}_{\left.p_{1}()_{1}\right)}^{\alpha, q_{(}(\cdot)}\left(\mathbb{R}^{n}\right)} \text {. }
$$

Let us now turn to estimate $\eta_{11}$. Noting that $x \in A_{j}$ and $j \leq k-2$, by the generalized Hölder's inequality and the Minkowski's inequality, we get

$$
\begin{aligned}
\left|T h_{j}(x)\right| & \leq \int_{A_{j}}\left|K(x, y) h_{j}(y)\right| \mathrm{d} y \\
& \leq C \int_{A_{j}}\left|h_{j}(y)\right| /|x-y|^{n} \mathrm{~d} y \\
& \leq C 2^{-k n} \int_{A_{j}}\left|h_{j}(y)\right| \mathrm{d} y \\
& \leq C 2^{-k n}\left\|h_{j}\right\|_{L^{1}\left(\mathbb{R}^{n}\right)} .
\end{aligned}
$$

By Lemmas 3.5-3.7 and the fact that $\left\|\frac{2^{j \alpha}\left|h \chi_{j}\right|}{\eta_{10}}\right\|_{L^{p_{1}(\cdot) q_{1}}} \leq 1$, we easily see that

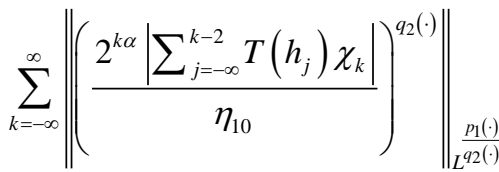

$$
\begin{aligned}
& \leq C \sum_{k=-\infty}^{\infty}\left\|\left(\frac{2^{k \alpha}\left|\sum_{j=-\infty}^{k-2} 2^{-k n}\left\|h_{j}\right\|_{L^{1}\left(\mathbb{R}^{n}\right)} \chi_{k}\right|}{\eta_{10}}\right)^{q_{2} \cdot(\cdot)}\right\|_{\frac{p_{1}(\cdot)}{q_{22} \cdot(\cdot)}}
\end{aligned}
$$$$
\leq C \sum_{k=-\infty}^{\infty}\left\|\left(\frac{2^{k \alpha}\left|\sum_{j==}^{\infty} 2^{-k n}\left\|h_{j}\right\|_{L^{1}\left(\mathbb{R}^{n}\right)} \chi_{k}\right|}{\eta_{10}}\right)_{L^{p^{1}(\cdot)}\left(\mathbb{R}^{n}\right)}\right\|^{\left(q_{2}^{2}\right)_{k}}
$$$$
\leq C \sum_{k=-\infty}^{\infty}\left(2^{k \alpha} \sum_{j=-\infty}^{k-2} 2^{-k n}\left\|\frac{h_{j}}{\eta_{10}}\right\|_{L^{p_{1}(\cdot)}\left(\mathbb{R}^{n}\right)}\left\|\chi_{B_{k}}\right\|_{L^{p_{1}(\cdot)}\left(\mathbb{R}^{n}\right)}\left\|\chi_{B_{j}}\right\|_{L^{p_{1}(\cdot)}\left(\mathbb{R}^{n}\right)}\right)^{\left(q^{2}\right)_{k}}
$$$$
\leq C \sum_{k=-\infty}^{\infty}\left(2^{k \alpha} \sum_{j=-\infty}^{k-2} 2^{-k n}\left\|\frac{h \chi_{j}}{\eta_{10}}\right\|_{L^{p_{1}(\cdot)}\left(\mathbb{R}^{n}\right)}\left(\left|B_{k}\right|\left\|\chi_{B_{k}}\right\|_{L^{p^{1}(\cdot)}\left(\mathbb{R}^{n}\right)}^{-1}\right)\left\|\chi_{B_{j}}\right\|_{L^{p_{1}}(\cdot)\left(\mathbb{R}^{n}\right)}\right)^{\left(q_{2}^{2}\right)_{k}}
$$$$
\leq C \sum_{k=-\infty}^{\infty}\left(2^{k \alpha} \sum_{j=-\infty}^{k-2} 2^{-j \alpha}\left\|\frac{||^{j \alpha} h \chi_{j} \|}{\eta_{10}}\right\|_{L^{p_{1}(\cdot)}\left(\mathbb{R}^{n}\right)} \frac{\left\|\chi_{B_{j}}\right\|_{L^{p_{1}(\cdot)}\left(\mathbb{R}^{n}\right)}}{\left\|\chi_{B_{k}}\right\|_{L^{p_{1}(\cdot)}\left(\mathbb{R}^{n}\right)}}\right)^{\left(q_{2}^{2}\right)_{k}}
$$$$
\leq C \sum_{k=-\infty}^{\infty}\left\{\sum_{j=-\infty}^{k-2} 2^{(k-j)\left(\alpha-n_{11}\right)}\left\|\left(\frac{\left|2^{j \alpha} h \chi_{j}\right|}{\eta_{10}}\right)^{q_{1}(\cdot)}\right\|_{\left.L^{p_{1}(\cdot) \cdot(\cdot) \cdot(\cdot)} \|_{\mathbb{R}^{n}}\right)}\right\}^{\frac{1}{\left.q_{1}\right)+}},
$$

where 


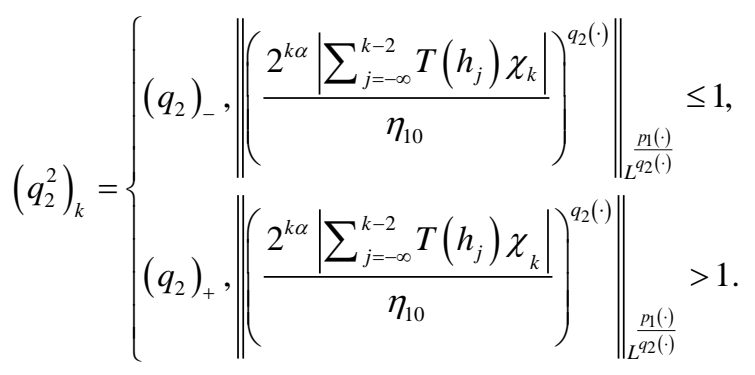

Therefore, if $\left(q_{1}\right)_{+}<1$ and $\left(p_{1}\right)_{+} \leq\left(p_{2}\right)_{-} \leq\left(q_{2}^{2}\right)_{k}$, we can get

$$
\begin{aligned}
& \sum_{k=-\infty}^{\infty}\left\|\left(\frac{2^{k \alpha}\left|\sum_{j=-\infty}^{k-2} T\left(h_{j}\right) \chi_{k}\right|}{\eta_{10}}\right)^{q_{2}(\cdot)}\right\|_{\substack{p_{1}(\cdot) \\
L^{2(\cdot)}}} \\
& \leq C\left\{\sum_{j=-\infty}^{\infty}\left\|\left(\frac{\left|2^{j \alpha} h \chi_{j}\right|}{\eta_{10}}\right)^{q_{1}(\cdot)}\right\|_{L^{p_{1}(\cdot) q_{1}(\cdot)}} \sum_{k=j+2}^{\infty} 2^{(k-j)\left(\alpha-n_{\left.\mu_{1}\right)}\right.}\right\}^{q_{*}} \\
& \leq C \text {, }
\end{aligned}
$$

where $q_{*}=\min _{k \in \mathbb{N}} \frac{\left(q_{2}^{1}\right)_{k}}{\left(q_{1}\right)_{+}}$.

If $\left(q_{1}\right)_{+} \geq 1$ and $\left(q_{2}^{2}\right)_{k} \geq\left(q_{2}\right)_{-} \geq\left(q_{2}\right)_{+} \geq 1$. By Remark 2.2 and applying the generalized Hölder's inequality, we obtain

$$
\begin{aligned}
& \sum_{k=-\infty}^{\infty}\left\|\left(\frac{2^{k \alpha}\left|\sum_{j=-\infty}^{k-2} T\left(h_{j}\right) \chi_{k}\right|}{\eta_{10}}\right)^{q_{2}(\cdot)}\right\|_{\frac{p_{1}(\cdot)}{L_{2_{2} \cdot(\cdot)}}} \\
& \leq C \sum_{k=-\infty}^{\infty}\left\{\sum_{j=-\infty}^{k-2}(k-j) 2^{(k-j)\left(\alpha-n_{11}\right)\left(q_{1}\right)_{+} / 2}\left\|\left(\frac{\left|2^{j \alpha} h \chi_{j}\right|}{\eta_{10}}\right)^{q_{1}(\cdot)}\right\|_{L^{p_{1}(\cdot) q_{1}(\cdot)}}\right\}^{\frac{\left(q_{2}^{2}\right)_{k}}{\left(q_{1}\right)^{+}}} \\
& \times\left(\sum_{j=-\infty}^{k-2} 2^{(k-j)\left(\alpha-n_{11}\right)\left(\left(q_{1}\right)_{+}\right)^{\prime} / 2}\right)^{\frac{\left(q_{2}^{2}\right)_{k}}{\left(\left(q_{1}\right)+\right)^{\prime}}} \\
& \leq C\left\{\sum_{j=-\infty}^{\infty}\left\|\left(\frac{\left|2^{j \alpha} h \chi_{j}\right|}{\eta_{10}}\right)^{q_{1}(\cdot)}\right\|_{L^{p_{1}(\cdot) q_{1}(\cdot)}} \sum_{k=j+2}^{\infty} 2^{(k-j)\left(\alpha-n_{11}\right)\left(q_{1}\right)_{+} / 2}\right\}^{q_{*}} \\
& \leq C \text {, }
\end{aligned}
$$

where $q_{*}=\min _{k \in \mathbb{N}} \frac{\left(q_{2}^{2}\right)_{k}}{\left(q_{1}\right)_{+}}$.

Hence, we see that

$$
\eta_{11} \leq C \eta_{10} \leq C\|h\|_{\dot{K}_{p_{1}(\cdot)}^{\alpha, q_{1}(\cdot)}\left(\mathbb{R}^{n}\right)} \cdot
$$

Finally, we estimate $\eta_{13}$. Noting that for each $x \in A_{j}$ and $j \geq k+2$, we have 


$$
\begin{aligned}
& \left|T h_{j}(x)\right| \leq \int_{A_{j}}\left|K(x, y) h_{j}(y)\right| \mathrm{d} y \leq C \int_{A_{j}}\left|h_{j}(y)\right| /|x-y|^{n} \mathrm{~d} y \leq C 2^{-j n}\left\|h_{j}\right\|_{L^{1}\left(\mathbb{R}^{n}\right)} . \\
& \text { By Lemma 3.7 and }\left\|\frac{2^{j \alpha}\left|h \chi_{j}\right|}{\eta_{10}}\right\|_{L^{p_{1}(\cdot) q_{1}(\cdot)}} \leq 1 \text {, we get } \\
& \sum_{k=-\infty}^{\infty}\left\|\left(\frac{2^{k \alpha}\left|\sum_{j=k+2}^{\infty} T\left(h_{j}\right) \chi_{k}\right|}{\eta_{10}}\right)^{q_{2}(\cdot)}\right\|_{\frac{p_{1}(\cdot)}{L_{22} \cdot(\cdot)}}
\end{aligned}
$$

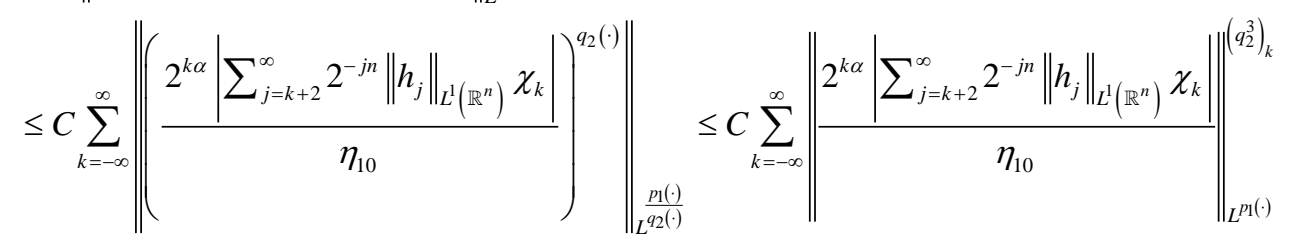

$$
\begin{aligned}
& \leq C \sum_{k=-\infty}^{\infty}\left(2^{k \alpha} \sum_{j=k+2}^{\infty} 2^{-j n}\left\|\frac{h_{j}}{\eta_{10}}\right\|_{L^{p_{1}(\cdot)}\left(\mathbb{R}^{n}\right)}\left\|\chi_{B_{k}}\right\|_{L^{p_{1}(\cdot)}\left(\mathbb{R}^{n}\right)}\left\|\chi_{B_{j}}\right\|_{L^{p^{1}(\cdot)}\left(\mathbb{R}^{n}\right)}\right)^{\left(q_{q^{3}}\right)_{k}} \\
& \leq C \sum_{k=-\infty}^{\infty}\left(2^{k \alpha} \sum_{j=k+2}^{\infty} 2^{-j n}\left\|\frac{h \chi_{j}}{\eta_{10}}\right\|_{L^{p^{1}(\cdot)}\left(\mathbb{R}^{n}\right)}\left(\left|B_{j}\right|\left\|\chi_{B_{j}}\right\|_{L^{p_{1}(\cdot)}\left(\mathbb{R}^{n}\right)}^{-1}\right)\left\|\chi_{B_{j}}\right\|_{L^{p_{1}(\cdot)}\left(\mathbb{R}^{n}\right)}\right)^{\left(q_{2}^{3}\right)_{k}} \\
& \leq C \sum_{k=-\infty}^{\infty}\left(2^{k \alpha} \sum_{j=k+2}^{\infty}\left\|\frac{h \chi_{j}}{\eta_{10}}\right\|_{L^{p_{1}(\cdot)}\left(\mathbb{R}^{n}\right)}\left\|\chi_{B_{j}}\right\|_{L^{p_{1}(\cdot)}\left(\mathbb{R}^{n}\right)}^{-1}\left\|\chi_{B_{j}}\right\|_{L^{p^{(} \cdot()}\left(\mathbb{R}^{n}\right)}\right)^{\left(q_{2}^{3}\right)_{k}} \\
& \leq C \sum_{k=-\infty}^{\infty}\left(2^{k \alpha} \sum_{j=k+2}^{\infty} 2^{-j \alpha}\left\|\frac{2^{j \alpha} h \chi_{j} \mid}{\eta_{10}}\right\|_{L^{p^{1} \cdot(\cdot)}\left(\mathbb{R}^{n}\right)} \frac{\left\|\chi_{B_{k}}\right\|_{L^{p_{1}(\cdot)}\left(\mathbb{R}^{n}\right)}}{\left\|\chi_{j_{k}}\right\|_{L^{p_{1}} \cdot(\cdot)}\left(\mathbb{R}^{n}\right)}\right)^{\left(q_{2}^{3}\right)_{k}} \\
& \leq C \sum_{k=-\infty}^{\infty}\left\{\sum_{j=k+2}^{\infty} 2^{(k-j)\left(\alpha+n_{12}\right)}\left\|\left(\frac{\left|2^{j \alpha} h \chi_{j}\right|}{\eta_{10}}\right)^{q_{1}(\cdot)}\right\|_{L^{p_{1}(\cdot) \cdot(\cdot)} \cdot \frac{1}{\left(q_{1}\right)^{+}}\left(\mathbb{R}^{n}\right)}\right\}^{\left(q_{2}^{3}\right)_{k}}
\end{aligned}
$$

where

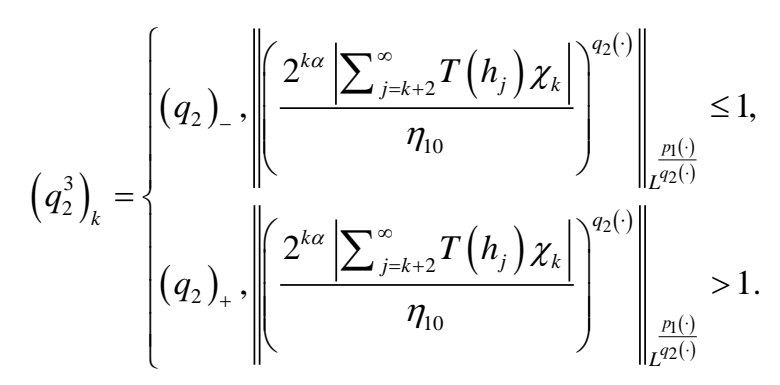

Then we have $\eta_{13} \leq C \eta_{10} \leq C\|h\|_{\dot{K}_{p_{1}(\cdot)}^{\alpha, q_{(} \cdot(\cdot)}\left(\mathbb{R}^{n}\right)}$, by using the same argument in $\eta_{11}$. Thus, we prove Theorem 4.1.

Theorem 4.2. Let $b \in \operatorname{BMO}\left(\mathbb{R}^{n}\right)$. Suppose that $p_{1}(\cdot) \in \mathcal{B}\left(\mathbb{R}^{n}\right), q_{1}(\cdot), q_{2}(\cdot) \in \mathcal{P}\left(\mathbb{R}^{n}\right) \quad$ with $\quad\left(q_{2}\right)_{-} \geq\left(q_{1}\right)_{+}$. If $-n u_{12}<\alpha<n u_{11}$ with $r_{11}, l_{12}$ as defined in lemma 3.5 , then the commutator $[b, T]$ is bounded from $\dot{K}_{p_{1}(\cdot)}^{\alpha, q_{2} \cdot(\cdot)}\left(\mathbb{R}^{n}\right)$ to $\dot{K}_{p_{1}(\cdot)}^{\alpha, q_{1}(\cdot)}\left(\mathbb{R}^{n}\right)$. 
Proof Let $h(x) \in \dot{K}_{p_{1}(\cdot)}^{\alpha, q_{1}(\cdot)}\left(\mathbb{R}^{n}\right), b \in \operatorname{BMO}\left(\mathbb{R}^{n}\right)$. We write

$$
h(x)=\sum_{j=-\infty}^{\infty} h(x) \chi_{j}=\sum_{j=-\infty}^{\infty} h_{j}(x)
$$

By virtue of the definition of $\dot{K}_{p(\cdot)}^{\alpha, q(\cdot)}\left(\mathbb{R}^{n}\right)$, we have

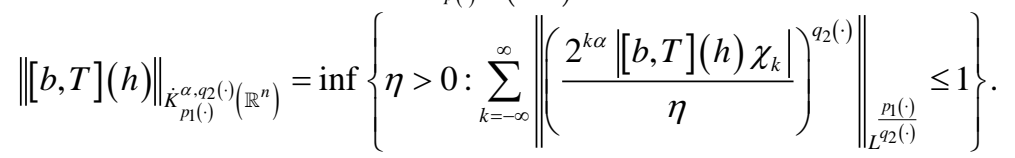

Since

$$
\begin{aligned}
& \left\|\left(\frac{2^{k \alpha}\left|[b, T](h) \chi_{k}\right|}{\eta}\right)^{q_{2}(\cdot)}\right\|_{\frac{p_{1}(\cdot)}{q_{2(\cdot)}}} \leq\left\|\left(\frac{2^{k \alpha}\left|\sum_{j=-\infty}^{k-2}[b, T]\left(h_{j}\right) \chi_{k}\right|}{\sum_{i=1}^{3} \eta_{2 i}}\right)^{q_{2}(\cdot)}\right\|_{L_{L^{2}}^{p_{1}(\cdot)}} \\
& \left.\leq \| \frac{2^{k \alpha}\left|\sum_{j=-\infty}^{\infty}[b, T]\left(h_{j}\right) \chi_{k}\right|}{\eta_{21}}\right)^{q_{2}(\cdot)}\left\|_{L_{L^{2}} \frac{p_{1}(\cdot)}{q_{2 \cdot} \cdot()}}+\right\|\left(\frac{2^{k \alpha}\left|\sum_{j=k-2}^{k+2}[b, T]\left(h_{j}\right) \chi_{k}\right|}{\eta_{22}}\right)^{q_{2}(\cdot)} \|_{\frac{p_{1}(\cdot)}{q_{q_{2} \cdot(\cdot)}}} \\
& +\left\|\left(\frac{2^{k \alpha}\left|\sum_{j=k+2}^{\infty}[b, T]\left(h_{j}\right) \chi_{k}\right|}{\eta_{23}}\right)^{q_{2}(\cdot)}\right\|_{\substack{p_{1}(\cdot) \\
q^{2(\cdot)}}} .
\end{aligned}
$$

Let

$$
\begin{gathered}
\eta_{21}=\left\|\left\{2^{k \alpha}\left|\sum_{j=-\infty}^{k-2}[b, T]\left(h_{j}\right) \chi_{k}\right|\right\}_{k=-\infty}^{\infty}\right\| \|_{\ell^{q_{2}}(\cdot)\left(L^{p_{1}(\cdot)}\right)}, \\
\eta_{22}=\left\|\left\{2^{k \alpha}\left|\sum_{j=k-2}^{k+2}[b, T]\left(h_{j}\right) \chi_{k}\right|\right\}_{k=-\infty}^{\infty}\right\| \|_{\ell^{q_{2}(\cdot)}\left(L^{p_{1}(\cdot)}\right)}, \\
\eta_{23}=\left\|\left\{2^{k \alpha}\left|\sum_{j=k+2}^{\infty}[b, T]\left(h_{j}\right) \chi_{k}\right|\right\}_{k=-\infty}^{\infty}\right\| \|_{\ell^{q_{2}(\cdot)}\left(L^{p_{1}(\cdot)}\right)},
\end{gathered}
$$

and

$$
\eta=\sum_{i=1}^{3} \eta_{2 i}
$$

Therefore, we can obtain

$$
\sum_{k=-\infty}^{\infty}\left\|\left(\frac{2^{k \alpha}\left|[b, T](h) \chi_{k}\right|}{\eta}\right)^{q_{2}(\cdot)}\right\|_{\frac{p_{1}(\cdot)}{q_{2}(\cdot)}} \leq C
$$

Thus it follows that,

$$
\|[b, T](h)\|_{\dot{K}_{p_{1}(\cdot)}^{\alpha, q(\cdot)}\left(\mathbb{R}^{n}\right)} \leq C \eta=C \sum_{i=1}^{3} \eta_{1 i} .
$$

Hence $\eta_{21}, \eta_{22}, \eta_{23} \leq C\|b\|_{\mathrm{BMO}\left(\mathbb{R}^{n}\right)}\|h\|_{\dot{K}_{p 1 \cdot(\cdot)}^{\alpha, q_{1}(\cdot)}\left(\mathbb{R}^{n}\right)}$. Denoting $\eta_{10}=C\|h\|_{\dot{K}_{p 1 .}^{\alpha, q_{1}(\cdot)}\left(\mathbb{R}^{n}\right)}$, firstly we estimate $\eta_{22}$ as in Theorem 4.1. Applying Lemma 3.3, we imme- diately arrive at 


$$
\sum_{k=-\infty}^{\infty}\left\|\left(\frac{2^{k \alpha}\left|\sum_{j=k-2}^{k+2}[b, T]\left(h_{j}\right) \chi_{k}\right|}{\eta_{10}\|b\|_{\mathrm{BMO}\left(\mathbb{R}^{n}\right)}}\right)^{q_{2}(\cdot)}\right\|_{\frac{p_{1}(\cdot)}{q^{2}(\cdot)}} \leq C .
$$

So we can get that

$$
\eta_{21} \leq C \eta_{10}\|b\|_{\mathrm{BMO}\left(\mathbb{R}^{n}\right)} \leq C\|b\|_{\mathrm{BMO}\left(\mathbb{R}^{n}\right)}\|h\|_{\dot{K}_{p_{1}(\cdot)}^{\alpha, q_{1} \cdot(\cdot)}\left(\mathbb{R}^{n}\right)} .
$$

Next we estimate $\eta_{21}$, Let $x \in A_{j}, j \leq k-2$.

$$
\begin{aligned}
& \left|[b, T] h_{j}\right| \leq \int_{A_{j}}\left|K(x, y)(b(x)-b(y)) h_{j}(y)\right| \mathrm{d} y \\
& \leq C \int_{A_{j}}\left|(b(x)-b(y)) h_{j}(y)\right| /|x-y|^{n} \mathrm{~d} y \\
& \leq C 2^{-n k}\left|b(x)-b_{B_{j}}\right| \int_{A_{j}}\left|h_{j}(y)\right| \mathrm{d} y+\int_{A_{j}}\left|b_{B_{j}}-b(y)\right|\left|h_{j}(y)\right| \mathrm{d} y \\
& \leq C 2^{-n k}\left|b(x)-b_{B_{j}}\right|\left\|h_{j}\right\|_{L^{1}\left(\mathbb{R}^{n}\right)}+\left\|b(\cdot)-\left(b_{B_{j}}\right) h_{j}\right\|_{L^{1}\left(\mathbb{R}^{n}\right)} .
\end{aligned}
$$

Thus, from Lemmas 3.4-3.7, We obtain that

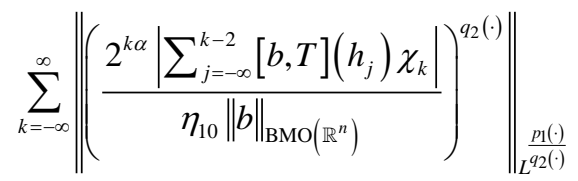

$$
\begin{aligned}
& \leq C \sum_{k=-\infty}^{\infty}\left\|\frac{2^{k \alpha}\left|\sum_{j=-\infty}^{k-2} 2^{-n k}\right| b(x)-b_{B_{j}} \mid\left\|h_{j}\right\|_{L^{1}\left(\mathbb{R}^{n}\right)} \chi_{k} \|}{\eta_{10}\|b\|_{\mathrm{BMO}\left(\mathbb{R}^{n}\right)}}\right\|_{q_{L^{2}}(\cdot)\left(\mathbb{R}^{n}\right)}
\end{aligned}
$$

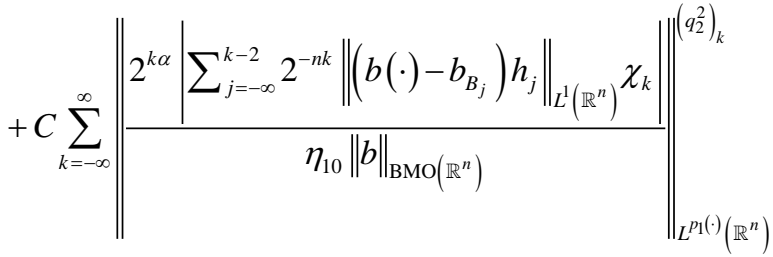

$$
\begin{aligned}
& \leq C \sum_{k=-\infty}^{\infty}\left(2^{k \alpha} \sum_{j=-\infty}^{k-2} 2^{-k n}\left\|\frac{h_{j}}{\eta_{10}}\right\|_{L^{p_{1}(\cdot)}\left(\mathbb{R}^{n}\right)}\|b\|_{\mathrm{BMO}^{-1}\left(\mathbb{R}^{n}\right)}^{-\|}\left\|\left(b(x)-b_{B_{j}}\right) \chi_{B_{j}}\right\|_{L^{p^{\prime}(\cdot)}\left(\mathbb{R}^{n}\right)}\left\|\chi_{B_{k}}\right\|_{L^{p_{1}(\cdot)}\left(\mathbb{R}^{n}\right)}\right)^{\left(q_{2}^{2}\right)_{k}} \\
& +C \sum_{k=-\infty}^{\infty}\left(2^{k \alpha} \sum_{j=-\infty}^{k-2} 2^{-k n}\left\|\frac{h_{j} \mid}{\eta_{10}}\right\|_{L^{p_{1}(\cdot)}\left(\mathbb{R}^{n}\right)}(k-j)\left\|\chi_{B_{k}}\right\|_{L^{p_{1}(\cdot)}\left(\mathbb{R}^{n}\right)}\left\|\chi_{B_{j}}\right\|_{L^{p^{\prime}(\cdot)}\left(\mathbb{R}^{n}\right)}\right)^{\left(q_{2}^{2}\right)_{k}} \\
& \leq C \sum_{k=-\infty}^{\infty}\left(2^{k \alpha} \sum_{j=-\infty}^{k-2} 2^{-k n}\left\|\frac{h_{j} \mid}{\eta_{10}}\right\|_{L^{p_{1}(\cdot)}\left(\mathbb{R}^{n}\right)}(k-j)\left\|\chi_{B_{k}}\right\|_{L^{p^{1} \cdot(\cdot)}\left(\mathbb{R}^{n}\right)}\left\|\chi_{B_{j}}\right\|_{L^{p^{\prime}(\cdot)}\left(\mathbb{R}^{n}\right)}\right)^{\left(q_{2}^{2}\right)_{k}} \\
& \leq C \sum_{k=-\infty}^{\infty}\left(2^{k \alpha} \sum_{j=-\infty}^{k-2}(k-j) 2^{-j \alpha}\left\|\frac{\left\|2^{j \alpha} h \chi_{j}\right\|}{\eta_{10}}\right\|_{L^{p_{1}(\cdot)}\left(\mathbb{R}^{n}\right)} \frac{\left\|\chi_{B_{j}}\right\|_{L^{p^{\prime}(\cdot)}\left(\mathbb{R}^{n}\right)}}{\left\|\chi_{B_{k}}\right\|_{L^{p^{\prime}(\cdot)}\left(\mathbb{R}^{n}\right)}}\right)^{\left(q_{2}^{2}\right)_{k}} .
\end{aligned}
$$


Therefore, we get

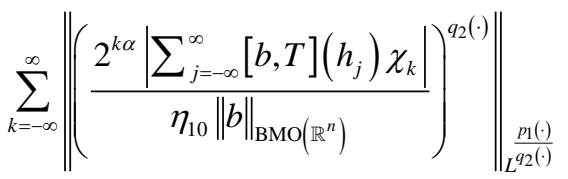

$$
\begin{aligned}
& \leq C \sum_{k=-\infty}^{\infty}\left\{\sum_{j=-\infty}^{k-2}(k-j) 2^{(k-j)\left(\alpha-n l_{11}\right)}\left\|\left(\frac{\left|2^{j \alpha} h \chi_{j}\right|}{\eta_{10}}\right)^{q_{1}(\cdot)}\right\|_{L^{p_{1}(\cdot) q_{1}(\cdot)\left(\mathbb{R}^{n}\right)}}^{\frac{1}{\left(q_{1}\right)+}}\right\}^{\left(q_{2}^{2}\right)_{k}},
\end{aligned}
$$

where

$$
\left(q_{2}^{2}\right)_{k}=\left\{\begin{array}{l}
\left(q_{2}\right)_{-},\left\|\left(\frac{2^{k \alpha}\left|\sum_{j=-\infty}^{k-2}[b, T]\left(h_{j}\right) \chi_{k}\right|}{\eta_{10}}\right)^{q_{2}(\cdot)}\right\|_{\frac{p_{1}(\cdot)}{q_{2}(\cdot)}} \leq 1, \\
\left.\left(q_{2}\right)_{+}, \| \frac{2^{k \alpha}\left|\sum_{j=-\infty}^{k-2}[b, T]\left(h_{j}\right) \chi_{k}\right|}{\eta_{10}}\right)^{q_{2}(\cdot)} \|_{\frac{p_{1}(\cdot)}{q_{q_{2}(\cdot)}}}>1 .
\end{array}\right.
$$

This, for $\left(q_{1}\right)_{+}<1,\left(p_{1}\right)_{+} \leq\left(p_{2}\right)_{-} \leq\left(q_{2}^{2}\right)_{k}$, along with Remark 2.2, tells us that

$$
\begin{aligned}
& \sum_{k=-\infty}^{\infty}\left\|\left(\frac{2^{k \alpha}\left|\sum_{j=-\infty}^{k-2}[b, T]\left(h_{j}\right) \chi_{k}\right|}{\eta_{10}\|b\|_{B M O}\left(\mathbb{R}^{n}\right)}\right)^{q_{2}(\cdot)}\right\|_{L^{p_{1}(\cdot)}} \\
& \leq C\left\{\sum_{j=-\infty}^{\infty}\left\|\left(\frac{\left|2^{j \alpha} h \chi_{j}\right|}{\eta_{10}}\right)^{q_{1}(\cdot)}\right\|_{L^{p_{1}(\cdot) q_{1}(\cdot)}} \sum_{k=j+2}^{\infty}(k-j) 2^{(k-j)\left(\alpha-n_{11}\right)}\right\}^{q_{*}} \leq C,
\end{aligned}
$$

where $q_{*}=\min _{k \in N} \frac{\left(q_{2}^{2}\right)_{k}}{\left(q_{1}\right)_{+}}$.

If $\left(q_{1}\right)_{+} \leq 1$, it is follows from Remark 2.2 and Hölder's inequality that

$$
\begin{aligned}
& \sum_{k=-\infty}^{\infty} \|\left(\frac{2^{k \alpha}\left|\sum_{j=-\infty}^{k-2}[b, T]\left(h_{j}\right) \chi_{k}\right|}{\left.\eta_{10}\|b\|_{\mathrm{BMO}\left(\mathbb{R}^{n}\right)}\right)^{q_{2}(\cdot)} \|_{\frac{p_{1}(\cdot)}{q_{2}(\cdot)}}}\right. \\
& \leq C \sum_{k=-\infty}^{\infty}\left\{\sum_{j=-\infty}^{k-2}(k-j) 2^{(k-j)\left(\alpha-n_{11}\right)\left(q_{1}\right)_{+} / 2}\left\|\left(\frac{\left|2^{j \alpha} h \chi_{j}\right|}{\eta_{10}}\right)^{q_{1}(\cdot)}\right\|_{L^{p_{1}(\cdot) q_{1}(\cdot)}}\right\}^{\frac{\left(q_{2}^{2}\right)_{k}}{\left(q_{1}\right)^{+}}}
\end{aligned}
$$$$
\times\left(\sum_{j=-\infty}^{k-2}(k-j) 2^{(k-j)\left(\alpha-n k_{11}\right)\left(\left(q_{1}\right)_{+}\right)^{\prime} / 2}\right)^{\frac{\left(q_{2}^{2}\right)_{k}}{\left(\left(q_{1}\right)+\right)^{\prime}}}
$$$$
\leq C\left\{\sum_{j=-\infty}^{\infty}\left\|\left(\frac{\left|2^{j \alpha} h \chi_{j}\right|}{\eta_{10}}\right)^{q_{1}(\cdot)}\right\|_{L^{p_{1}(\cdot) \cdot\left(q_{1}(\cdot)\right.}} \sum_{k=j+2}^{\infty}(k-j) 2^{(k-j)\left(\alpha-n n_{11}\right)\left(q_{1}\right)_{+} / 2}\right\}^{q_{*}}
$$

$\leq C$, 
where $q_{*}=\min _{k \in N} \frac{\left(q_{2}^{2}\right)_{k}}{\left(q_{1}\right)_{+}}$.

This implies that

$$
\eta_{21} \leq C \eta_{10}\|b\|_{\mathrm{BMO}\left(\mathbb{R}^{n}\right)} \leq C\|b\|_{\mathrm{BMO}\left(\mathbb{R}^{n}\right)}\|h\|_{\dot{K}_{p_{1}(\cdot)}^{\alpha, q_{1}(\cdot)}\left(\mathbb{R}^{n}\right)} .
$$

Finally we estimate $\eta_{23}$, for any $x \in A_{j}, j \geq k+2$, by the same way to argument in $\eta_{21}$, we obtain that

$$
\begin{aligned}
\left|[b, T] h_{j}\right| & \leq \int_{A_{j}}\left|K(x, y)(b(x)-b(y)) h_{j}(y)\right| \mathrm{d} y \\
& \leq C \int_{A_{j}}\left|(b(x)-b(y)) h_{j}(y)\right| /|x-y|^{n} \mathrm{~d} y \\
& \leq C 2^{-n j}\left|b(x)-b_{B_{k}}\right| \int_{A_{j}}\left|h_{j}(y)\right| \mathrm{d} y+\int_{A_{j}}\left|b_{B_{k}}-b(y)\right|\left|h_{j}(y)\right| \mathrm{d} y \\
& \leq C 2^{-n j}\left|b(x)-b_{B_{j}}\right|\left\|h_{j}\right\|_{L^{1}\left(\mathbb{R}^{n}\right)}+\left\|b(\cdot)-\left(b_{B_{j}}\right) h_{j}\right\|_{L^{1}\left(\mathbb{R}^{n}\right)},
\end{aligned}
$$

and

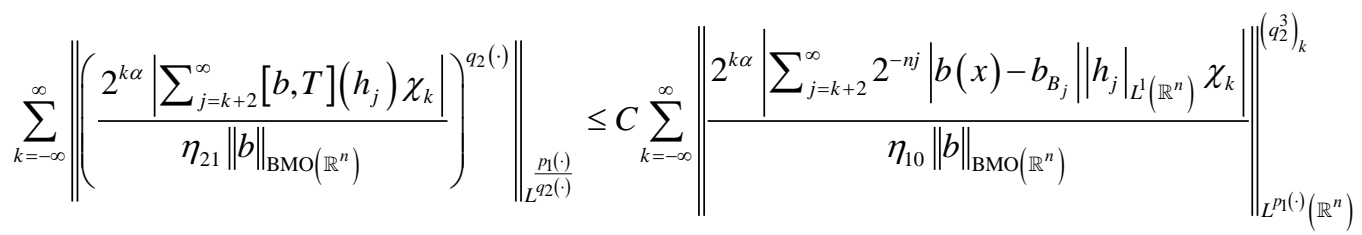

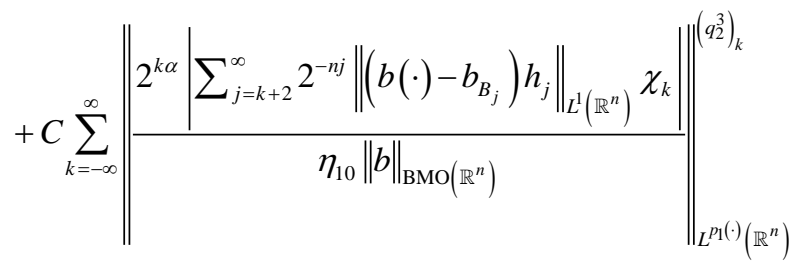

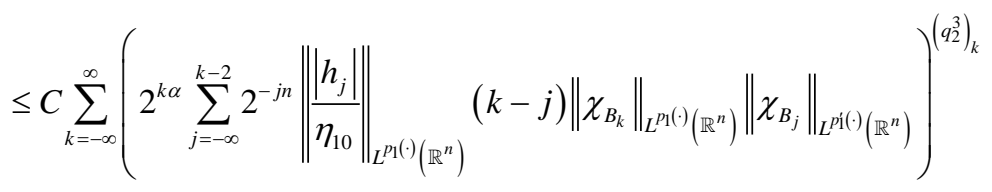

$$
\begin{aligned}
& \leq C \sum_{k=-\infty}^{\infty}\left(2^{k \alpha} \sum_{j=-\infty}^{k-2}(j-k) 2^{-j \alpha}\left\|\frac{\left|2^{j \alpha} h \chi_{j}\right|}{\eta_{10}}\right\|_{L^{p^{1}(\cdot)}\left(\mathbb{R}^{n}\right)} \frac{\left\|\chi_{B_{k}}\right\|_{L^{p_{1}(\cdot)}\left(\mathbb{R}^{n}\right)}}{\left\|\chi_{B_{j}}\right\|_{L^{p_{1}(\cdot)}\left(\mathbb{R}^{n}\right)}}\right)^{\left(q_{2}^{3}\right)_{k}} \\
& \leq C \sum_{k=-\infty}^{\infty}\left\{\sum_{j=-\infty}^{k-2}(j-k) 2^{(k-j)\left(\alpha+n n_{12}\right)}\left\|\left(\frac{\left|2^{j \alpha} h \chi_{j}\right|}{\eta_{10}}\right)^{q_{1}(\cdot)}\right\|_{L^{p_{1}(\cdot) \cdot(\cdot) \cdot(\cdot)}\left(\mathbb{R}^{n}\right)}^{\left(\frac{1}{\left(q_{1}\right)+}\right.}\right\}^{\left(q_{2}^{3}\right)_{k}}
\end{aligned}
$$

where

$$
\left(q_{2}^{3}\right)_{k}=\left\{\begin{array}{l}
\left(q_{2}\right)_{-},\left\|\left(\frac{2^{k \alpha}\left|\sum_{j=-\infty}^{k-2}[b, T]\left(h_{j}\right) \chi_{k}\right|}{\eta_{10}}\right)^{q_{2}(\cdot)}\right\|_{\frac{p_{1}(\cdot)}{q_{2} \cdot()}} \leq 1, \\
\left(q_{2}\right)_{+},\left\|\left(\frac{2^{k \alpha}\left|\sum_{j=-\infty}^{k-2}[b, T]\left(h_{j}\right) \chi_{k}\right|}{\eta_{10}}\right)^{q_{2}(\cdot)}\right\|_{\frac{p_{1}(\cdot)}{q_{2(\cdot)}}}>1 .
\end{array}\right.
$$


Hence, we arrive at that $\eta_{23} \leq C \eta_{10}\|b\|_{\mathrm{BMO}\left(\mathbb{R}^{n}\right)} \leq C\|b\|_{\mathrm{BMO}\left(\mathbb{R}^{n}\right)}\|h\|_{\dot{K}_{p_{1}(\cdot)}^{\alpha, q_{1}(\cdot)}\left(\mathbb{R}^{n}\right)}$ by the similar argument in the proof Theorem 4.1.

This completes the proof of Theorem 4.2.

\section{Acknowledgements}

This paper is supported by National Natural Foundation of China (Grant No. 11561062).

\section{References}

[1] Calderón, A. and Zygmund, A. (1956) On Singular Integrals. American Journal of Mathematics, 78, 289-309. https://doi.org/10.2307/2372517

[2] Calderón, A. and Zygmund, A. (1978) On Singular Integral with Variable Kernels. Applied Analysis, 7, 221-238. https://doi.org/10.1080/00036817808839193

[3] Jouné, J.-L. (1983) Calderón-Zygmund Operators, Pseudo-Differential Operators and the Cauchy Integral of Calderón. In: Lecture Notes in Math, Vol. 994, Springer-Verlag, Berlin, Heidelberg.

[4] Coifman, R., Rochberg, R. and Weiss, G. (1976) Factorization Theorems for Hardy Spaces in Several Variables. Annals of Mathematics, 103, 611-635.

https://doi.org/10.2307/1970954

[5] Kováčik, O. and Rákosník, J. (1991) On Spaces $L^{p(x)}$ and $W^{k, p(x)}$. Czechoslovak Mathematical Journal, 41, 592-618.

[6] Diening, L. and Ruicka, M. (2003) Calderón-Zygmund Operators on Generalized Lebesgue Spaces $L^{p(\cdot)}$ and Problems Related to Fluid Dynamics. Journal für die Reine und Angewandte Mathematik, 563, 197-220. https://doi.org/10.1515/crll.2003.081

[7] Chen, Y., Levine, S. and Rao, M. (2006) Variable Exponent, Linear Growth Functionals in Image Restoration. SIAM Journal on Applied Mathematics, 66, 1383 1406. https://doi.org/10.1137/050624522

[8] Li, F., Li, Z. and Pi, L. (2010) Variable Exponent Functionals in Image Restoration. Applied Mathematics and Computation, 216, 870-882.

[9] Harjulehto, P., Hästö, P., Latvala, V. and Toivanen, O. (2013) Critical Variable Exponent Functionals in Image Restoration. Applied Mathematics Letters, 26, 56-60.

[10] Izuki, M. (2009) Herz and Amalgam Spaces with Variable Exponent, the Haar Wavelets and Greediness of the Wavelet System. East Journal on Approximations, $15,87-109$.

[11] Izuki, M. (2010) Boundedness of Commutators on Herz Spaces with Variable Exponent. Rendiconti del Circolo Matematico di Palermo, 59, 199-213. https://doi.org/10.1007/s12215-010-0015-1

[12] Izuki, M. (2010) Fractional Integrals on Herz-Morrey Spaces with Variable Exponent. Hiroshima Mathematical Journal, 40, 343-355.

[13] Wang, L. and Tao, S. (2014) Boundedness of Littlewood-Paley Operators and Their Commutators on Herz-Morrey Spaces with Variable Exponent. Journal of Inequalities and Applications, 2014, 227. https://doi.org/10.1186/1029-242x-2014-227

[14] Wang, L. and Tao, S. (2015) Parameterized Littlewood-Paley Operators and Their Commutators on Lebegue Spaces with Variable Exponent. Analysis in Theory and Applications, 31, 13-24.

[15] Tan, J. and Liu, Z. (2015) Some Boundedness of Homogeneous Fractional Integrals 
on Variable Exponent Function Spaces. ACTA Mathematics Science (Chinese Series), 58, 310-320.

[16] Omer, A., Afif, A. and Tao, S. (2016) The Boundedness for Commutators of Calderón-Zygmund Operator on Herz-Tybe Hardy Spaces with Variable Exponent. Journal of Applied Mathematics and Physics, 4, 1157-1167. https://doi.org/10.4236/jamp.2016.46120

[17] Wang, L. and Tao, S. (2016) Parameterized Littlewood-Paley Operators and Their Commutators on Herz Spaces with Variable Exponents. Turkish Journal of Mathematics, 40, 122-145. https://doi.org/10.3906/mat-1412-52

[18] Cuz-Uribe, D., Fiorenza, A., Martell, J.M. and Perez, C. (2006) The Boundedness of Classical Operators on Variable $L^{p}$ Spaces. Annales Academiae Scientiarum Fennicae-Mathe-matica, 31, 239-264.

[19] Almeida, A., Hasanov, J. and Samko, S. (2008) Maximal and Potential Operators in Variable Exponent Morrey Spaces. Georgian Mathematical Journal, 15, 195-208.

[20] Cruz-Uribe, D. and Fiorenza, A. (2013) Variable Lebesgue Spaces: Foundations and Harmonic Analysis. In: Applied and Numerical Harmonic Analysis, Springer, New York.

[21] Diening, L. (2005) Maximal Function on Musielak-Orlicz Spaces and Generalized Lebesgue Spaces. Bulletin des Sciences Mathématiques, 129, 657-700. https://doi.org/10.1016/j.bulsci.2003.10.003

Submit or recommend next manuscript to SCIRP and we will provide best service for you:

Accepting pre-submission inquiries through Email, Facebook, LinkedIn, Twitter, etc. A wide selection of journals (inclusive of 9 subjects, more than 200 journals) Providing 24-hour high-quality service

User-friendly online submission system

Fair and swift peer-review system

Efficient typesetting and proofreading procedure

Display of the result of downloads and visits, as well as the number of cited articles Maximum dissemination of your research work

Submit your manuscript at: http://papersubmission.scirp.org/

Or contact am@scirp.org 